\title{
Escuela y alumnado: (Des)encuentros en la relación con el saber
}

\author{
School and Students: Encounters and Disagreements in Relation with Knowledge
}

Escolas e estudantes: (Des) encontros em relação ao saber

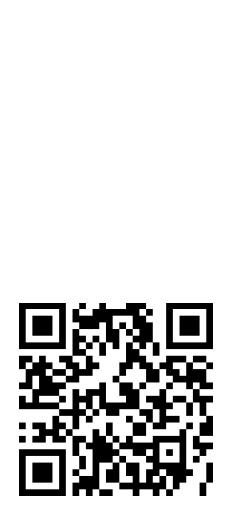

\author{
Soledad Vercellino ${ }^{1}$ \\ Centro Interdisciplinario de Estudios en Derechos \\ Viedma, Argentina \\ svercellino@unrn.edu.ar \\ Nora Liliana Tarruella ${ }^{2}$ \\ Universidad Nacional \\ Centro Regional Zona Atlántica \\ Viedma, Argentina \\ ntarruella@hotmail.com
}

Romina Alejandra van den Heuvel ${ }^{3}$

Universidad Nacional

Centro Regional Zoina Atlántica

Viednam Argentina

rominavdh@hotmail.co

Rocío Belén Andrade 4

Universidad Nacional

Centro Regional Zoina Atlántica

Viedma, Argentina

rociobelen11@hotmail.com

\footnotetext{
${ }^{1}$ Licenciada en Psicopedagogía Universidad Nacional del Comahue, Magíster en Sociedad e Instituciones, Universidad Nacional de San Luis. Profesora Adjunta Regular del Área Teoría y Metodología de la Investigación Universidad Nacional de Río Negro, asistente de docencia regular en la misma área en la Universidad Nacional del Comahue. Directora del Centro Interdisciplinario de Estudios en Derechos, Inclusión y Sociedad de la Universidad Nacional de Río Negro. Posee publicaciones en revistas nacionales e internacionales, un libro de autoría propia, varios capítulos de libros en coautoría y una importante formación de recursos humanos en investigación.

${ }^{2}$ Licenciada y Profesora en Psicopedagogía. Maestranda en Psicoanálisis de la Universidad Nacional de Mar del Plata. Actualmente Docente e investigadora del CURZA, Universidad Nacional del Comahue. Ha sido profesora regular en el área de ciencias de la educación y psicología de un instituto de formación docente, directora de Nivel Superior en la provincia de Río Negro, y participante en la elaboración de diseños curriculares de formación docente y planes de estudios de profesorados en la universidad.

${ }^{3}$ Licenciada y Profesora en Psicopedagogía. Ejerce hace 3 años como ayudante de docencia en la Universidad Nacional del Comahue - Centro Regional Zona Atlántica. Participa en la misma institución en investigación, hace 4 años, como integrante alumna, becaria alumna y actualmente como docente. Posee publicaciones en revistas nacionales e internacionales, en coautoría y derivadas de la investigación, además de presentaciones en congresos nacionales.

${ }^{4}$ Profesora en Psicopedagogía de la Universidad Nacional del Comahue-CURZA-. Actualmente es docente en el nivel medio y universitario. Desde el año 2008 viene desempeñándose como técnica en el Área de la Modalidad de Educación Intercultural Bilingüe del Ministerio de Educación y DD. HH. Dicha labor implica un trabajo de articulación con organizaciones, comunidades originarias, autoridades y referentes del pueblo Mapuche y organismos gubernamentales. En el año 20013 comenzó a participar en el campo de la investigación, como integrante alumna, luego como pasante graduada. Actualmente se incorporó como colaboradora en el PI 40-C-450 denominado "Ser indígena en el Valle Inferior del río Negro: Aproximación teórica". Universidad Nacional de Río Negro. Dirigido por el Dr. Luciano Prates (Universidad Nacional de la Plata- UNLP) y codirigido por el Dr. Javier Serrano de la Universidad de Río Negro.
} 
doi: http://dx.doi.org/10.15359/ree.21-3.10

URL: http://www.una.ac.cr/educare

CORREO: educare@una.cr

\author{
Mariana Andrea Guerreiro 5 \\ Universidad Nacional \\ Centro Regional Zoina Atlántica \\ Viedma, Argentina \\ marianaandreaguerreiro@gmail.com \\ Cristina Irigoyen ${ }^{6}$ \\ Universidad Nacional \\ Centro Regional Zoina Atlántica \\ Viedma, Argentina \\ crisuni54@hotmail.com \\ Lidia Mónica Cardinale ${ }^{7}$ \\ Universidad Nacional \\ Centro Regional Zona Atlántica \\ Viedma, Argentina \\ Imcardin@yahoo.com.ar
}

Recibido 2 de setiembre de 2016 • Corregido 22 de junio de 2017 • Aceptado 6 de agosto de 2017
Received 2 de setiembre de 2016 • Revised 22 de junio de 2017 • Accepted 6 de agosto de 2017
Recebido 2 de setiembre de 2016 • Revisado 22 de junio de 2017 • Aprovado 6 de agosto de 2017

Resumen: El siguiente artículo presenta la experiencia de campo y algunos resultados preliminares de una investigación llevada a cabo en el marco del Proyecto de Investigación "La constitución de la relación con el saber en los alumnos del último ciclo del nivel primario y primero del nivel medio" (Universidad Nacional del Comahue. Departamento de Psicopedagogía, 2015), desarrollado en el Departamento Psicopedagogía del Centro Regional Zona Atlántica de la Universidad Nacional del Comahue. En continuidad con estudios de las ciencias humanas que abordan la relación con el saber de los sujetos, y siguiendo los aportes teóricos de Charlot (2008), esta indagación se propuso analizar

\footnotetext{
${ }^{5}$ Licenciada en Psicopedagogía por el Centro Universitario Regional Zona Atlántica de la Universidad Nacional del Comahue (Viedma, Río Negro, Argentina). He desarrollado una investigación, en el marco de mi tesis de licenciatura, titulada: "La relación de sujetos adultos con el saber corporal en el marco de la enseñanza y el aprendizaje del método pilates". Me he desempeñado como pasante alumna en el Proyecto de Investigación cuyos resultados se exponen en el presente artículo.

${ }^{6}$ Profesora de Actividades Prácticas, Licenciada en Psicopedagogía, Profesora en Psicopedagogía, y en la actualidad, maestranda comenzando la Tesis de la "Maestría Educación en Entornos Virtuales de enseñanza-aprendizaje". Docente universitaria en ejercicio en las cátedras, Metodología de las Materias Psicopedagógicas, Practica Docente y Didáctica General, en la Universidad Nacional del Comahue, sede CURZA, Río NegroArgentina; integro proyectos de investigación. Fui docente de educación primaria, de nivel medio, nivel especial y nivel laboral e integrante de los ETAP (Equipos Técnicos de Apoyo Pedagógico); me desempeñé como coordinadora de la Escuela Terapeútica "Escribiendo mi Futuro" y clínica privada, cargos en los que me encuentro jubilada. Poseo publicaciones en autoría y coautoría derivadas de la investigación.

${ }^{7}$ Psicopedagoga. Especialista en Ciencias Sociales con mención en Curriculum y Practicas Escolares. En etapa de presentación de Tesis de la Maestría en Educación con orientación en Ciencias Sociales. Profesora regular en el área "Psicológica-Pedagógica" orientación "Pedagógico Didáctica" con 18 años de antigüedad docente y 19 años como investigadora del CURZA de la Universidad Nacional del Comahue. Posee publicaciones en revistas nacionales e internacionales. Es autora de capítulo de libros editados por EDUCO (Democratización de la Universidad. Investigaciones y experiencias sobre el acceso y la permanencia de los/las estudiantes) y Miño y Dávila (Innovaciones educativas. Entre las políticas públicas y la práctica educativa), derivados de los trabajos de investigación. También es autora de otras publicaciones realizadas en el marco del PROMERE (UNCo) del cual fue miembro integrante durante los años 2004 a 2012.
}

2 Soledad Vercellino, Nora Liliana Tarruella, Romina Alejandra van den Heuvel, Rocío Belén Andrade, Mariana Andrea Guerreiro, Cristina Irigoyen y Lidia Mónica Cardinale

Los artículos de la Revista Electrónica Educare del Centro de Investigación y Docencia en Educación de la Universidad Nacional, Costa Rica, se comparten bajo términos de la Licencia Creative Commons: Reconocimiento, № Comercial, Sin Obra Derivada 3.0 Costa Rica. Las autorizaciones adicionales a las aquí delimitadas se pueden obtener en el correo: educare@una.cr 
los procesos secundarios de constitución de la relación del alumnado con el saber, focalizando en alumnos y alumnas que culminan el nivel primario y que inician el nivel medio en escuelas públicas de la provincia de Río Negro. Identificando y problematizando, específicamente, los sentidos que este alumnado otorga a los saberes escolares, y más en general, a la escuela y a sus trayectos en ella: pasados y futuros. La opción metodológica fue de carácter cualitativa, hermenéutico interpretativa. Se implementó un taller de acompañamiento psicopedagógico que consistió en distintos encuentros, desarrollados dentro del horario escolar, organizados, cada uno, en torno a un nudo problemático particular y articulado a una actividad-producción que buscó recuperar el discurso del alumnado. A partir de las producciones (escritas, orales, gráficas, dramáticas), se pesquisaron los objetos-saberes de los que se han apropiado, las actividades que saben dominar y formas relacionales aprehendidas, a través del análisis de las distintas dimensiones de la relación con el saber: en un primer momento la epistémica, y luego la social e identitaria. Como resultados, encontramos que el alumnado refirió a distintas figuras del aprender (Charlot, 2008), dando espacial relevancia a los modos de relacionarse. A su vez, desde la escuela, se enfatiza en la aprehensión del saber relacional pero como saberobjeto. Respecto al saber-escribir, se visualiza que su escritura sigue una normatividad diferente a la propuesta en la escuela, que podríamos denominar estándar. Finalmente, cuando aluden a los lugares en los que aprenden, si bien mencionan distintos espacios extraescolares y extrafamiliares, la escuela y la familia siguen apareciendo como sitios e instituciones privilegiadas para el aprendizaje.

Palabras clave: Relación con el saber; saberes escolares; figuras del aprender; pasaje a nivel medio.

Abstract: The following article presents some preliminary results of a study conducted under the research project "The constitution of the relation with knowledge in students of the last cycle of primary education and the first one of secondary level" (National University of Comahue, Department of Psychopedagogy, 2015), implemented by the Department of Psychopedagogy at the Regional Center Atlantic Area, National University of Comahue, Argentina. In continuity with studies of human sciences that address the relation with knowledge of the subjects, and following Charlot's (2008) theoretical contributions, this study aimed to analyze the secondary processes of establishing the relation of students with knowledge, focusing on students who culminate the primary education and start secondary level in public schools in Rio Negro Province. Identifying and problematizing specifically the meanings these students give to school knowledge, and more generally, to school and their past and future trajectories in it. The research methodology was qualitative, hermeneutical and interpretative. We implemented a psycho-pedagogical accompanying workshop; it consisted in various meetings carried out during school hours, each one organized around a particular problem and linked to a production activity- sought, to recover the students' discourse. On the basis of written, oral, graphic and dramatic productions, we researched the objects of knowledge, the activities the students mastered, and the relational forms acquired. As a result, we found that students reported different figures of learning (Charlot, 2008), giving special relevance to the ways of relating. In turn, from school, the apprehension of knowledge is emphasized but as knowledge-relational object. Regarding the know-writing, writing follows different rules to those proposed at school that we might call standard. Finally, when you refer to the places where they learn, even though they mention different spaces to school and family, these two spaces continue to be privileged sites and institutions for learning.

Keywords: Relation to knowledge; school knowledge; figures of learning; transition to secondary level; high school level. 
doi: http://dx.doi.org/10.15359/ree.21-3.10

URL: http://www.una.ac.cr/educare

CORREO: educare@una.cr

\begin{abstract}
Resumo: $O$ seguinte artigo apresenta a experiência de campo e alguns resultados preliminares de uma investigação realizada no âmbito do projeto de investigação: "O estabelecimento da relação com o conhecimento, nos alunos de educação infantil e do ensino fundamental" (Universidade Nacional de Comahue. Departamento de Psicopedagogia, 2015), desenvolvido no Departamento de Psicopedagogia do centro regional Atlântico da Universidade Nacional de Comahue. Em continuidade com estudos das ciências humanas que abordam a relação com o conhecimento dos sujeitos, e seguindo as contribuições teóricas de Charlot (2008), esta pesquisa faz uma análise dos processos secundários que estabelecem a relação dos alunos com o saber, enfatizando aqueles estudantes que terminaram o nível primário e iniciaram o ensino fundamental - nos colégios públicos da província do Rio Negro. Identificar e problematizar, especificamente, o significado que os estudantes dão ao conhecimento escolar, e de forma más geral a escola, e os caminhos percorridos dentro dela: passados e futuros. O enfoque metodológico utilizado foi qualitativo e de interpretação hermenêutica. Realizou-se uma oficina de acompanhamento psicopedagógico, organizado em várias reuniões aplicadas durante o horário escolar, de forma que cada reunião girava em torno a uma problemática particular, articulada a uma atividade-produção que procurava recuperar a participação de cada estudante. A partir de produções (escritas, oral, gráfica, dramática), o núcleo da pesquisa foi conhecer os objetos-saberes que foram apropriados, os trabalhos que sabem realizar, as formas de entrosamento aprendidas, através da analises das diferentes dimensões da relação com o saber: em primeiro lugar epistêmico, e em seguida, social e de construção identitária. Os resultados encontrados enfatizaram a opinião dos estudantes, quando estes manifestaram os diferentes papeis da aprendizagem (Charlot, 2008), enfatizando as varias maneiras de relacionamento social. Por sua vez, na escola, o destaque está na preocupação do saber relacional, mas como objeto de conhecimento. Sobre o saber-escrito, percebe-se que a escritura segue uma proposta diferente a da escola, que poderíamos chamar de regulamentos padrão. Finalmente, quando se referem aos lugares onde se aprende, embora existam vários espaços extra institucionais e extrafamiliares, tanto a escola como a família continuam sendo lugares privilegiados para a aprendizagem.
\end{abstract}

Palavras-chave: relação com o saber; conhecimento escolar; papéis da aprendizagem; mudança a outro nível educativo.

\title{
Introducción
}

En las dos últimas décadas la situación educativa latinoamericana se caracterizó por la sanción de leyes que aumentaron la cantidad de años de la educación obligatoria. En Argentina, en 2014, prácticamente la totalidad de los niños y niñas de 5 a 14 años estaban escolarizados. En el caso de la región Patagónica, la tasa de escolarización de la población entre 6 y 14 años ascendía a más del 99\% y en la franja de 15 a 17 años, descendía diez puntos decimales, ubicándose en el 89, 73 \% (Poggi, 2015).

Ahora bien, como contrapartida se advierte que la universalización en el acceso a la escuela primaria y media no implica necesariamente el ejercicio pleno del derecho a la educación. Así, los datos de rendimiento interno de los sistemas educativos de la región indican que la escuela

$4 \quad$ Soledad Vercellino, Nora Liliana Tarruella, Romina Alejandra van den Heuvel, Rocío Belén Andrade, Mariana Andrea Guerreiro, Cristina Irigoyen y Lidia Mónica Cardinale

Los artículos de la Revista Electrónica Educare del Centro de Investigación y Docencia en Educación de la Universidad Nacional, Costa Rica, se comparten bajo términos de la Licencia Creative Commons: Reconocimiento, No Comercial, Sin Obra Derivada 3.0 Costa Rica. Las autorizaciones adicionales a las aquí delimitadas se pueden obtener en el correo: educare@una.cr 
no logra que una significativa cantidad del alumnado complete su educación obligatoria en los tiempos esperados. El porcentaje de alumnos y alumnas con dos o más años de retraso escolar en la primaria o secundaria ascendía, por ejemplo, en nuestra Patagonia, al 16,02\%, con el guarismo más alto entre las regiones del país (Poggi, 2015). La sobreedad alcanza al finalizar el nivel primario el $16 \%$ en Río Negro, y asciende al $20 \%$ en el total país y al finalizar el primer año de la secundaria alcanza el 37\% en ambas jurisdicciones (Ministerio de Educación y Deportes. DINIEE, 2015). La repitencia, especialmente en los primeros años del nivel secundario, es muy elevada, alcanza, en Río Negro, un 33\% en primer año (Scasso, 2012).

Sectores académicos y organismos internacionales advierten que los sistemas educativos deben enfrentar aún un desafío:

[Que los alumnos y las alumnas] logren apropiarse de los saberes que ese sistema se propone transmitir; ... la preocupación por la mejora de las trayectorias escolares y de los aprendizajes en ellas logrados se sitúa en las prioridades de la actual agenda de política educativa de numerosos países de la región. (Poggi, 2015, pp. 11, 13)

Otros denuncian que el alumnado establece con la escuela y con sus aprendizajes relaciones de baja intensidad (Kessler, 2007), es decir, no logra "conectarse" con los aprendizajes escolares, sentirse "atrapado" por ellos; encuentra dificultades para darle un sentido a estos y para articularlos con otros aspectos de su vida ${ }^{8}$.

En el marco de esta compleja problemática nuestra investigación focaliza en el aprendizaje escolar y sus vicisitudes. A tal fin seguiremos profundizando el estudio de una noción que ha comenzado a expandirse en el campo de las ciencias humanas, y que resulta fértila la comprensión psicopedagógica: la relación del alumnado con el saber (Vercellino, 2014; Vercellino, van den Heuvel, Guerreiro, 2014).

Desde hace unos años venimos problematizando los distintos desplazamientos teóricos que ha tenido la noción de relación con el saber (Vercellino, 2015; van den Heuvel y Vercellino, 2015; van den Heuvel, 2016) y evaluando las posibilidades teóricas de esta misma para el análisis psicopedagógico de los aprendizajes escolares (Andrade, 2016) y no escolares (Guerreiro, 2016). Asimismo, comenzamos una investigación exploratoria sobre la relación con el saber que han construido alumnado de nivel primario-

Estas indagaciones se han realizado en el marco de dos proyectos de investigación financiados por la Universidad Nacional del Comahue. Se trata del proyecto "Relación de los

\footnotetext{
${ }_{8}^{8}$ Parafraseamos aquí la categoría de "escolaridad de baja intensidad aportada por Kessler (2007). Así como este investigador retoma el concepto de ciudadanía de baja intensidad, y lo lleva al ámbito escolar, nos permitimos mudarlo ahora al ámbito más singular del aprendizaje para dar cuenta del fenómeno que nos interesa investigar.
} 
doi: http://dx.doi.org/10.15359/ree.21-3.10

URL: http://www.una.ac.cr/educare

CORREO: educare@una.cr

alumnos con el saber y formato escolar. Investigación teórica y empírica con alumnos de escuelas primarias de la ciudad de Viedma, Río Negro. 2013-2014" (Universidad Nacional del Comahue. Departamento de Psicopedagogía, 2013), el Proyecto "La constitución de la relación con el saber en los alumnos del último ciclo del nivel primario y primero del nivel medio. Viedma, Río Negro. 2015-2016" (Universidad Nacional del Comahue. Departamento de Psicopedagogía, 2015). Este artículo presenta resultados de esta última investigación.

Como ya se ha desarrollado ampliamente en Vercellino (2015), los estudios sobre la relación con el saber se inician en Francia, en el campo del psicoanálisis, en la década del 60. Será a finales de los 80 y durante los años 90 , en los que aparecerán las obras que son los pilares sobre las que, posteriormente, se desarrolla la investigación que trata la relación con el saber. Dichas obras provienen de tres grupos de investigación consolidados: el grupo creado por Jacky Beillerot y continuado por Nicole Mosconi y Claudine Blanchard -Laville en el Centre de Recherche Education et Formation (CREF), de la Universidad Paris X-Nanterre, quienes desde diferentes teorías psicoanalíticas intentan esclarecer las "nociones de saber y de relación con el saber. Por eso [recurren] tanto a Sigmund Freud como a Melanie Klein o a Jacques Lacan, o también a Wilfred Bion y Donald Winnicott como a Cornélius Castoriadis" (Beillerot, BlanchardLaville y Mosconi, 1998, p. 4).

También en Francia, el equipo de investigación "Educación, socialización y colectividades locales" (ESCOL), dirigido inicialmente por el sociólogo Bernard Charlot en el marco del Departamento de Ciencias del Educación de la Universidad París VIII, Saint Denis, desde una corriente crítica y antropológica de la sociología de la educación (Anderson-Levitt, 2012) comienza a desarrollar este concepto en la década de 1970 y lo convierte, en los años 1980 y 1990, en un tema de investigación empírica (Rochex, 2004).

[Ese equipo] desarrolla investigaciones sobre la relación con el saber y la relación con la escuela de jóvenes que frecuentan establecimientos escolares situados en la periferia. Una primera investigación se ocupó del college y, en menor medida, de las escuelas primarias (Merlot, Bautier y Rochex, 1992). El equipo se interesó de inmediato en los liceos: Elizabeth Bautier y Jean-Ives Rochex trabajaron sobre los liceos generales y técnicos, y [Charlot] sobre los lycees professionnnels. (Charlot, 2008, p. 17)

Finalmente, el concepto de relación con el saber también ha encontrado terreno fértil en el campo de la didáctica en general, y en la didáctica de la matemática en particular, con los trabajos de Chevallard (2000) en el Institut de recherches sur l'enseignement des mathématiques (IREM) de la Universidad Aix-Marseille (Vercellino, 2015). Otros grupos investigadores que hemos consultado afiliados a esta línea son Venturini, Calmettes, Amade-Escot y Terrise (2007) y Thériault, Bader y Ndong Angoe (2013). Este enfoque focaliza en la relación que un sujeto o

6 Soledad Vercellino, Nora Liliana Tarruella, Romina Alejandra van den Heuvel, Rocío Belén Andrade, Mariana Andrea Guerreiro, Cristina Irigoyen y Lidia Mónica Cardinale

Los artículos de la Revista Electrónica Educare del Centro de Investigación y Docencia en Educación de la Universidad Nacional, Costa Rica, se comparten bajo términos de la Licencia Creative Commons: Reconocimiento, No Comercial, Sin Obra Derivada 3.0 Costa Rica. Las autorizaciones adicionales a las aquí delimitadas se pueden obtener en el correo: educare@una.cr 
una institución mantiene con el saber disciplinar, escolar o científico. Desde esta perspectiva se sostendrá que la relación institucional con el saber constituye y modifica la relación personal con el saber (Kalali, 2007; Pouliot, Bader y Therriault, 2010), por ello, la relación con el saber nunca es definitiva, las formas de relación con el saber de las instituciones pueden entonces entrar en conflicto con la propia relación personal del sujeto con el saber (Kalali 2007).

Advertimos:

Más allá de su inscripción en una u otra escuela, las investigaciones coinciden en que la relación de un sujeto con el saber se constituye en dos procesos vitales, uno primario, vinculado a las primeras relaciones que el niño o la niña desarrollan en el ámbito familiar, que imprime ciertas características a dicha relación; son marcas -más o menos posibilitadoras- que los procesos de subjetivación dejan en la forma en que el sujeto se relaciona con el saber. $Y$ un proceso secundario, vinculado al tránsito por instituciones exogámicas -las del sistema educativo, las de la inserción laboral- que con sus propios reservorios de significaciones, lógicas de funcionamiento y demandas hacia el sujeto, parecerían ser oportunidad u ocasión para conmover, modificar o reforzar dicha relación. (Vercellino, 2015, p. 76)

El presente artículo presenta la experiencia de campo y algunos resultados preliminares de una investigación en curso, busca profundizar el análisis de los procesos secundarios de constitución de la relación del alumnado con el saber, focalizando en estudiantes que culminan el nivel primario y que inician el nivel medio en escuelas públicas de la provincia de Río Negro. Específicamente se busca identificar y problematizar los sentidos que el alumnado otorga a los saberes escolares, y más en general, a la escuela y a sus trayectos en ella: pasados y futuros.

Siguiendo a Charlot (2008), partimos de un concepto amplio de relación con el saber, entendido como "... la relación con el mundo, con el otro y consigo mismo de un sujeto confrontado a la necesidad de aprender. [Como] el conjunto (organizado) de las relaciones que un sujeto mantiene con todo lo que se refiere al 'aprender' y al saber" (Charlot, 2008, p. 131).

Analizaremos tal relación pesquisando, en primer lugar, la relación epistémica con el saber, es decir, explorando con qué tipos de saberes (saberes objetos, actividades, modos de relación) el alumnado interactúa y que procesos de aprendizajes se dan con este (objetivaciónapropiación, dominio, etc.). Luego, consideraremos que aprender tiene sentido en referencia "a la historia del sujeto, ... sus relaciones con los otros, a la imagen que tiene de sí mismo y a aquella que quiere dar a los otros" (Charlot, 2008, p. 117). Es decir, atenderemos la dimensión social e identitaria de la relación con el saber. 
doi: http://dx.doi.org/10.15359/ree.21-3.10

URL: http://www.una.ac.cr/educare

CORREO: educare@una.cr

\section{Metodología}

En concordancia con el enfoque conceptual explicitado, se optó por una estrategia de investigación decaráctercualitativa porentenderqueestamismanos permitiríaabordarla complejidad de los procesos subjetivos e institucionales en los que se constituye la relación del alumnado con el saber. Esta decisión supuso una opción epistemológica de un enfoque hermenéutico/ interpretativo, antes que hipotético deductivo. Es decir, se priorizó la producción de categorías interpretativas a partir de los datos y no la verificación o corroboración de hipótesis previas.

La indagación, localizada en la ciudad de Viedma, tomó como unidades de análisis a estudiantes que culminaban el nivel primario en una escuela incorporada desde hace seis años en la modalidad de jornada extendida. La selección del contexto del trabajo de campo y de las unidades de análisis fue estratégica y teórica (Glaser y Strauss, 2009). El criterio teórico nos orientó a trabajar con el alumnado que está finalizando la escuela primaria a partir del supuesto de que el próximo pasaje al nivel medio podía constituirse en oportunidad para narrarse como aprendientes, y desde allí pesquisar los procesos secundarios de constitución de la relación del alumnado con el saber. Estratégicamente se optó por una escuela en la que habíamos desarrollado otros trabajos, lo que facilitó el acceso y la anuencia de los actores escolares a participar de la investigación.

La muestra quedó conformada 26 alumnos y alumnas del tercer ciclo del nivel primario (distribuidos en dos grupos-taller: un grupo de $7^{\mathrm{mo}}$ y otro grupo de $6^{\text {to }} \mathrm{y} 7^{\text {to }}$ ).

En la selección de la estrategia de recolección de datos se buscó recuperar el discurso del propio alumnado, apostando a su capacidad de historizar y dar sentido a su tránsito escolar y su vínculo con el saber. Se diseñó como técnica un taller de acompañamiento psicopedagógico denominado "De la primaria a la secundaria, un tiempo para pensar ese pasaje". Consistió en 4 encuentros, de 80 minutos cada uno, desarrollados dentro del horario escolar, organizados, cada uno, en torno a un nudo problemático particular y articulado a una actividad- producción.

En el primer encuentro el eje de las producciones se orientó a la presentación del taller, de sus participantes y de la problemática del pasaje al nivel medio. En el segundo taller se abordó la dimensión epistémica y social (Charlot, 2008) de la problemática del pasaje y se articuló en torno a estas preguntas: ¿Qué de lo que sé, de lo que he aprendido, será importante en el nivel medio (conocimientos, actividades, formas de relacionarme)? ¿Dónde y con quiénes aprendo? Para indagar las figuras del aprender se diseñó un instrumento semiestructurado en el que se le consultaba al alumnado sobre qué harían en determinadas situaciones que podrían afrontar en el nivel medio. Por ejemplo: ¿Si el profesor o profesora de historia explica un tema y no entiendo? ¿Si tuvieras que elegir hacer un trabajo sobre un tema que te gusta, que elegirías? ¿Si consideras que un profesor o una profesora fue injusto o injusta con vos, qué harías? El instrumento contenía ciertas opciones preestablecidas y la posibilidad de incluir otras, tal como consta en la Figura 1:

8 Soledad Vercellino, Nora Liliana Tarruella, Romina Alejandra van den Heuvel, Rocío Belén Andrade, Mariana Andrea Guerreiro, Cristina Irigoyen y Lidia Mónica Cardinale

Los artículos de la Revista Electrónica Educare del Centro de Investigación y Docencia en Educación de la Universidad Nacional, Costa Rica, se comparten bajo términos de la Licencia Creative Commons: Reconocimiento, № Comercial, Sin Obra Derivada 3.0 Costa Rica. Las autorizaciones adicionales a las aquí delimitadas se pueden obtener en el correo: educare@una.cr 
doi: http://dx.doi.org/10.15359/ree.21-3.10

URL: http://www.una.ac.cr/educare

CORREO: educare@una.cr

¿Qué harías?

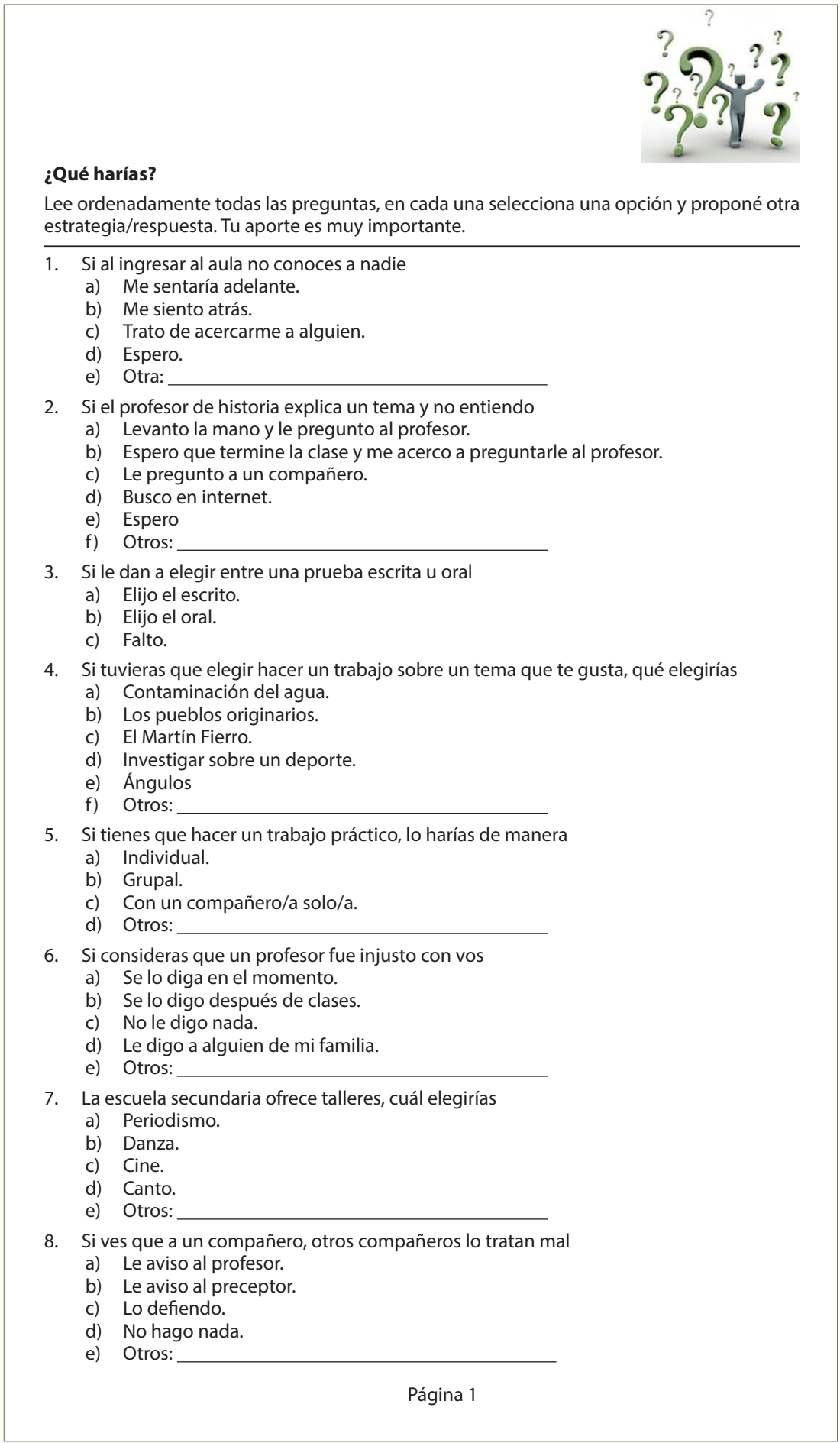

Figura 1: Instrumento administrado al alumnado para indagar la dimensión epistémica de la relación con el saber. 
doi: http://dx.doi.org/10.15359/ree.21-3.10

URL: http://www.una.ac.cr/educare

CORREO: educare@una.cr

En el tercer encuentro se analizó la dimensión identitaria de la problemática ¿Cómo soy como aprendiente? Las actividades-producciones de cada encuentro fueron variadas: trabajos escritos, gráficos, representaciones, juegos y producciones virtuales. El último taller fue de cierre y consistió en la presentación por parte de las personas coordinadoras de la memoria de las producciones desarrolladas durante los encuentros, lo que permitió el acceso a multiplicidad de voces, dibujos, imágenes y palabras del grupo, una reconstrucción de los proyectos, deseos y temores en palabras para ser leídas, que revelan su relación con el saber. Concebimos la devolución como una oportunidad para poner de relieve los aspectos subjetivos de la totalidad y cada estudiante, un nuevo texto antes inexistente para el estudiantado, asimismo una ocasión para elaborar la pérdida de lo ya vivido en la escuela primaria y para diseñar un modo de habitar la secundaria, un espacio por-venir. En la Tabla 1 se sintetiza el contenido de los talleres realizados.

Tabla 1: Síntesis de los talleres

\begin{tabular}{ll}
\hline Encuentro & Contenidos \\
\hline Primer encuentro & Presentación del taller, de sus participantes y de la problemática del pasaje al nivel medio. \\
Segundo encuentro & $\begin{array}{l}\text { Nudo problemático: Dimensión epistémica de la problemática del pasaje: ¿Qué de las } \\
\text { cosas que sé serán importantes en el nivel medio (conocimientos, actividades, formas } \\
\text { de relacionarme)? } \\
\text { Dimensión social de la problemática: ¿Dónde y con quiénes aprendo? }\end{array}$ \\
Tercer encuentro & $\begin{array}{l}\text { Dimensión identitaria de la problemática ¿Cómo soy como aprendiente? } \\
\text { Cuarto encuentro }\end{array}$ \\
\hline
\end{tabular}

\section{Resultados}

La escuela en la que se desarrolló esta investigación es una institución de casi 50 años ubicada en la zona sur de la ciudad, cuya población ha presentado históricamente altos índices de necesidades básicas insatisfechas 9 . Por ello, desde el año 1970 ha sido incluida en experiencias de ampliación de la jornada escolar (jornada completa, jornada extendida ${ }^{10}$ ), con la incorporación de talleres (laborales, inicialmente, y temáticos, luego) en el contra turno. La matrícula de la escuela ha ido variando en la medida en que se fueron creando escuelas en zonas de su radio de influencia (por el emplazamiento de nuevos barrios de vivienda), llegando

\footnotetext{
${ }^{9}$ Necesidades básicas insatisfechas (NBI) es un método directo para identificar carencias críticas en una población y caracterizar la pobreza. Usualmente utiliza indicadores directamente relacionados con cuatro áreas de necesidades básicas de las personas (vivienda, servicios sanitarios, educación básica e ingreso mínimo), disponibles en los censos de población y vivienda.

${ }^{10}$ Sobre la implementación de estas estrategias de extensión de jornada escolar para atender a poblaciones denominadas 'vulnerables' o en 'riesgo escolar', ver: Vercellino (2013), Cardinale (2015), Cardinale y Abel (2013).
}

10 Soledad Vercellino, Nora Liliana Tarruella, Romina Alejandra van den Heuvel, Rocío Belén Andrade, Mariana Andrea Guerreiro, Cristina Irigoyen y Lidia Mónica Cardinale

Los artículos de la Revista Electrónica Educare del Centro de Investigación y Docencia en Educación de la Universidad Nacional, Costa Rica, se comparten bajo términos de la Licencia Creative Commons: Reconocimiento, No Comercial, Sin Obra Derivada 3.0 Costa Rica. Las autorizaciones adicionales a las aquí delimitadas se pueden obtener en el correo: educare@una.cr 
a tener 400 alumnos a principio de la década de los 90. En el año 2015 tiene una población de 173 estudiantes distribuidos en 14 secciones de grado. Siete estudiantes reciben apoyo para su inclusión educativa y un $18 \%$ registra sobreedad (Gobierno de Río Negro. Ministerio de Educación y Derechos Humanos, 2015). Posee edificio propio que fue objeto de refacción y acondicionamiento; no obstante, aún faltan puertas en algunas aulas, la sala de informática consiste en un pequeño lugar, construido con otro fin, abarrotado de mobiliario, en varias aulas se observan bancos escolares en desuso. Si bien ofrece desayuno, almuerzo y merienda, no dispone del espacio de comedor, se utiliza para tal fin el salón de usos múltiples (que también es patio de juegos y actividades físicas) y las aulas (Cardinale, 2016).

Al ser consultados por sus aprendizajes, el alumnado participante de esta investigación refirió distintas figuras del aprender (Charlot, 2008): aludieron a objetos-saberes de los que se han apropiado, a actividades que saben dominar y a la aprehensión de ciertas formas relacionales.

Su repertorio de objetos-saber incluía: "extraterrestres, las plantas, contaminación y capa de ozono, animales, investigar en ciencias sociales, en medio ambiente o sobre vida marina", investigar sobre un deporte, la contaminación del agua, el Martín Fierro, ángulos, los pueblos originarios. También destacaban a la escuela como el lugar que podría permitirles dominar ciertas actividades: "canto, taller de mecánica, instrumentos, deportes, dibujo artístico, tecnología, carpintería, hojalatería, ingles", cine, danza, periodismo.

Pero, fundamentalmente, dieron cuenta de sus aprendizajes de formas de relacionarse, aprendizajes sobre cómo dominar ciertas situaciones que se dan en la vida escolar: cómo conducirse si no se conoce a nadie ("juego con ellos, busco un conocido, le digo al profesor que me presente, trato de hacer amigos, me siento solo/a, me hago a un lado", me siento atrás, trato de acercarme a alguien); qué hacer si no entiendo la explicación del profesorado ("me levanto y le pregunto al profe, le pregunto a mi familia, trato de recordar lo que aprendí, busco en un libro, le pregunto a mi compañero, le pregunto a mi hermano'), o si ven que a un compañero o compañera, otros alumnos o alumnas les tratan mal ("culparlo, hacerlo sentir bien, lo abrazo así no se siente mal", lo defiendo), que grupalmente o con otro compañero o compañera es la mejor forma de resolver tareas escolares, etc.

Como advierte la investigación en la temática (Charlot, 2008; Zambrano, 2013, entre otros), frente a estos objetos, a estas actividades, a estos dispositivos y formas, el aprendizaje no pasa por los mismos procesos. Saber sobre deportes o sobre la contaminación del agua, supone una actividad de apropiación de un saber cuya existencia se asienta en objetos, lugares -materiales o virtuales-, mediado por personas. Un saber que en la cultura occidental es fundamentalmente escrito"1. Charlot (2008) denomina objetivación-denominación "al proceso epistémico que constituye, en un mismo movimiento, un saber -objeto y un sujeto consciente de haberse

\footnotetext{
${ }^{11}$ No es el caso de otras culturas, como la del Pueblo Mapuche, cuyo acervo de saberes es primordialmente de tipo oral. Sobre la temática se han expresados autores como Quilaqueo, Quintriqueo y Cárdenas (2005), Quintriqueo y Torres (2012) y el trabajo desarrollado en el marco de este PI, Andrade (2016).
} 
doi: http://dx.doi.org/10.15359/ree.21-3.10

URL: http://www.una.ac.cr/educare

CORREO: educare@una.cr

apropiado de un tal saber" (p. 111). Otros teóricos de la relación con el saber, como Beillerot et al. (1998) denominarán a este saber objetivado, como 'conocimiento', "discursos denotativos ... exteriores para un sujeto ... algo dado (posible), algo sabido" (Beillerot, et al., 1998, p. 66).

El saber en tanto saber-objeto es lo que en las concepciones ordinarias se entiende como conjuntos de conocimientos. Estos son "inventariados, clasificados, acumulados, yacen en los libros o en las bases de datos. Los verbos que se emplean para hablar de los saberes son del orden del haber: se posee, se adquiere, se tiene tal o cual saber" (Beillerot et al., 1998, p. 31). En tal sentido, resulta interesante destacar cómo, en el caso estudiado, el escenario escolar se encuentra despoblado de los soportes típicos de esos saberes: en nuestra estadía en la escuela en la que desarrollamos la investigación, no vimos libros circulando en las aulas, ni afiches o pósters en las paredes, sí en varias oportunidades el recurso audiovisual de ciertos programas educativos de la televisión pública apareció como soporte.

Asimismo, el alumnado dio cuenta de otras relaciones con el saber, en tanto dominio de actividad. Resulta difícil para la investigación pesquisar este tipo de aprendizajes, pues el dominio de una actividad se inscribe en el cuerpo (Charlot, 2008; Guerreiro, 2016), siendo muy difícil nominar, objetivar el producto de ese aprendizaje. Este no es aquí separable de la actividad: se aprende a danzar, cantar, dibujar, practicar ciertos deportes, a tocar la guitarra, a andar en skate. Se puede, sin embargo, adoptar una posición reflexiva (metacognitiva) y designar, nombrar dichas actividades, no obstante ello, el aprendizaje de esos enunciados no es equivalente al aprendizaje de la actividad misma. Aprender a cantar, como dominio de la actividad, no implica aprender canto, es decir, dar cuenta de un conjunto de enunciados, en general normativos, que constituyen ese saber objeto.

Históricamente, las principales actividades que la escuela permitía dominar fueron la lectoescritura y el cálculo. Quienes participaron de este trabajo dicen preferir la expresión escrita a la oral. La investigación permitió advertir que la escritura que produjeron en el marco de los talleres (ver fragmentos de escritura que aparecen más adelante) sigue otra normatividad, distinta a la lengua estándar.

Por otro lado, algunos dominan saberes tecnológicos: saben poner en marcha la netbooks del gabinete de la escuela, utilizan sitios de juegos o redes sociales. Les resulta difícil dar cuenta del saber objeto tecnológico, es decir, explicitar reflexivamente los procedimientos que realizan para desarrollar tales actividades, así como también transferir esos conocimientos tecnológicos a otras actividades vinculadas, como es el caso del uso del correo electrónico, del procesador de texto, etc.

Finalmente, también reconocen que en el ámbito escolar aprenden a comportarse con solidaridad, responsabilidad, a no pelearse, a ayudar a las otras personas. Aprenden a dominar las relaciones intersubjetivas en situación: la relación de sí consigo mismo o misma, la relación de sí con otros sujetos (pares, personas adultas, familiares yno familiares, individuos conocidosodesconocidos), y la relación consigo a través de la relación con otros u otras y viceversa. Estos aprendizajes tampoco

12 Soledad Vercellino, Nora Liliana Tarruella, Romina Alejandra van den Heuvel, Rocío Belén Andrade, Mariana Andrea Guerreiro, Cristina Irigoyen y Lidia Mónica Cardinale

Los artículos de la Revista Electrónica Educare del Centro de Investigación y Docencia en Educación de la Universidad Nacional, Costa Rica, se comparten bajo términos de la Licencia Creative Commons: Reconocimiento, № Comercial, Sin Obra Derivada 3.0 Costa Rica. Las autorizaciones adicionales a las aquí delimitadas se pueden obtener en el correo: educare@una.cr 
pueden ser autonomizados, separados de la relación en situación. Sin embargo, aquí también se puede adoptar una posición reflexiva y designar la relación: decirse actuando, valorar(se) y valorar a las demás personas según como se conduzcan. El alumnado dice que sabe:

Respetar a los mayores y a los compañeros

NO PEGARLE AUNA MUJER

Hacer amigos; no pegarles a las mujeres

Respetar Las Normas De Comvivencias!

Repetarr A Los Mayores Etc!!

alludar a mitio en el taller; a escuchar y a respetar ${ }^{12}$ (alumnos de 6 to y 7 mo grado)

Se trata de enunciados -en general normativos- de contenido ético-político, sobre el buen conducirse y la relación con los demás sujetos. En nuestro pasaje por la institución escolar advertimos cómo esta insiste con el esfuerzo de transformar en saber objeto, a los saberes relacionales. En los momentos comunitarios, en las clases propiamente dicha, en las ofertas de talleres (titulado"Aprender a convivir-Afinando emociones") el saber objeto relacional aparece como un saber privilegiado: gran parte del tiempo escolar se destina a enunciar, nombrar, prescribir la forma de habitar la escena escolar: callado, en silencio, sin correr, no se grita, sentado, sin la gorra, respetar al personal docente, etc. Se trata de un saber objeto no planificado, pero que opera como condición preparatoria para la enseñanza escolar. Sin embargo, la apropiación de tales enunciados no es, necesariamente, equivalente al aprendizaje de la relación misma, en situación y en acto. Por ello, las personas adultas de la institución no dejan de denunciar que los saberes relacionales que domina el alumnado se contradicen o no se condicen con los saberes objeto relacionales que la escuela enuncia y propone.

\section{La relación con el saber: Su dimensión identitaria y sociaı}

Ahora bien, aprender es desplegar una actividad en situación: en un lugar, en un momento, en diversas condiciones de tiempo, con la ayuda de personas. El estudiantado que participó de esta investigación refiere a lugares de estatutos diferentes en los cuales aprende. Una parte alude a lugares de la vida (la casa, el barrio, la calle), otra a quienes se dedican a una actividad específica que no es la educación (el taller mecánico de un familiar, por ejemplo), pero la mayoría identificará a la escuela como el espacio específico en el que aprender.

En nuestra estancia en la escuela advertimos que los espacios escolares objetivos, es decir, aquellos que la arquitectura escolar configura según ciertas funciones específicas, ciertas personas y actividades que albergarán, pierden consistencia por efecto de las prácticas efectivas que se desarrollan en ellos: el aula deja de ser un poco aula cuando ingresa la comida, cuando la ausencia de puertas no (de)limita un adentro y un afuera; cuando sus paredes no registran ninguna marca de

${ }^{12}$ Estos son extractos de escrituras del alumnado que participó en esta investigación. Nos interesa transcribirlos de manera fiel a los originales, pues ponen de manifiesto lo señalado más arriba en relación con la forma en que dominan la escritura. 
doi: http://dx.doi.org/10.15359/ree.21-3.10

URL: http://www.una.ac.cr/educare

CORREO: educare@una.cr

las producciones estudiantiles, ni de qué curso se trata; cuando en ese espacio se apilan pupitres en desuso. El aula es un poco aula, un poco comedor, un poco pasillo, un poco depósito, un poco sala de reuniones. La pérdida de consistencia del espacio objetivo se traduce en una indiferenciación de los lugares: la reunión con el padre o madre se mantiene en el umbral del aula y la conversación se vuelve pública; el momento de estudio en el aula es invadido por las risas y gritos de otros grupos jugando en el pasillo; es difícil reconocer el lugar de la dirección pues no está identificado, todo el tiempo circula gente, no hay símbolos que le den un lugar jerarquizado ni distintivo. La forma de habitar los distintos lugares escolares (aula, pasillo, patio, dirección, sala de maestros, sala de informática) ha generado una fisonomía que (des)acredita su identidad (Camilloni, 2001).

Como advierte Mar (2009):

... la escuela es el reflejo de políticas educativas, modelos de gobierno, modelos e ideales pedagógicos, experimentos de innovación, ejercicios de ladrillo y cemento. Así, en la lectura de las escuelas se podrían notar varias capas de teoría pedagógica, algunas escuelas de arquitectura, varias disposiciones de organismos internacionales sobre la educación y muchos vacíos sobre la relación entre ladrillo y saber, espacio y hábitat, propuestas pedagógicas y formas de llevarlas a cabo y, sobre todo, vacíos en la comprensión de los sujetos de la educación en el espacio. (p. 8)

En otro orden, familia y escuela siguen operando como instituciones de referencia para el aprendizaje. En el caso de estudiantes que participaron de esta investigación, no enuncian otros referentes para su aprender.

Cuando se les consulta sobre "Una persona que les ha enseñado mucho" señalan:

Cecilia, vivi,

SEÑO SESILIA,

La Seño Cecilia Y El Profe Rolando,

Susana, Cecilia,

con la que mas aprendi es mi profe pablo,

la seño cesi,

seño sesi

profe rolando,

rolan y Cecilia,

a seño: Cecilia

MI PAPAY MI MAMA,

mi mama me ha enseñado muchas cosas,

Mi mamá,

MI TIO ALEX MENSEÑO MUCHAS COSAS (alumnos de 6to y 7 mo grado)

14 Soledad Vercellino, Nora Liliana Tarruella, Romina Alejandra van den Heuvel, Rocío Belén Andrade, Mariana Andrea Guerreiro, Cristina Irigoyen y Lidia Mónica Cardinale

Los artículos de la Revista Electrónica Educare del Centro de Investigación y Docencia en Educación de la Universidad Nacional, Costa Rica, se comparten bajo términos de la Licencia Creative Commons: Reconocimiento, № Comercial, Sin Obra Derivada 3.0 Costa Rica. Las autorizaciones adicionales a las aquí delimitadas se pueden obtener en el correo: educare@una.cr 
Consultado el grupo por sus expectativas sobre la escuela secundaria, mayoritariamente refiere a esta como el lugar en el que aprender saberes- objetos, enunciados en forma precisa en algunos casos (física, guitarra), y vaga en otros (cosas nuevas, muchas cosas, etc.). En sus palabras:

que me enseñen cosas nuevas

Aprenda mucho

Que me enseñan muchas cosas para cuando sea grande tenga un buen trabajo

Que me enseñen a tocar la guitarra

Que tenga laboratorio

Que sea para aprender

Que men ceñen ficica porque voi acer arquitecto

Aprender nuevas cosas

Que sea buena con todos mis compañeros

Divertida,

Que seacul

Espero que los compañeros sean buenos

Que estén mis compañeros

Que sea linda como la escuela primaria (alumnos de 6to y 7mo grado)

No obstante ello, al ser consultados por lo que les gusta mucho hacer, aparecen otras actividades que implican aprendizajes. Transcribimos algunas de ellas:

Jugar al footboll

Carate y futbol

Jugar al futbol

Estar con mis amigas y escuchar mucica

Jugar al fudbol

El futbol, hanbbol y bati sodbol

Jugar al futbol

Jugar al futbol

Jugar al futbol

El rap

Jugar al partido y salir a cazar

El fucboll y la mecanica

El futbol

Organisar proyectos de salidas.

Trabajar en grupo, trabajar con el compañero

Leer y escribir y cantar y fudbol (alumnos de 6to y 7 mo grado) 
doi: http://dx.doi.org/10.15359/ree.21-3.10

URL: http://www.una.ac.cr/educare

CORREO: educare@una.cr

Deportes, música, canto, etc., se trata de saberes-hacer, de dominios de actividad que no son referenciados con la escuela. Charlot (2008) plantea que una actividad es interesante para el sujeto en tanto converge una relación con el mundo, consigo y una relación con el otro. Parece ser que el fútbol -principalmente- resulta interesante porque es convocante en "mi mundo" con "otros", en una práctica no escolar. Es un saber hacer de gran valor en su comunidad, y una vía de reconocimiento, de identificación (con un club), un significante que hace lazo social (actividad grupal en ambos géneros).

Ahora bien, toda relación con el saber es también relación consigo: a través del 'aprender' "está siempre en juego la construcción de sí y su eco reflexivo, la imagen de si" (Charlot, 2008, p. 117) Se trata de la dimensión identitaria de esa relación: aprender tiene sentido en referencia a la historia del sujeto, sus expectativas, sus antecedentes, sus relaciones con las otras personas, a la imagen que tiene de sí y a aquella que quiere dar a los demás individuos.

¿Cómo se conciben como aprendientes? ¿Cómo se imaginan como aprendientes en el contexto de la escuela secundaria? El estudiantado representa en sus producciones (afiches, dramatizaciones) esta situación futura con los siguientes enunciados:

Me miran mal

Me siento muy mal

Conozco personas

Chicos y chicas que molestan

Llorando, tengo miedo.

Tengo mucha vergüenza

Una chica tímida: todos se ríen de ella.

Estoy nervioso

Me siento mal

Tener miedo.

Contento

Más difícil la tarea

Las pautas de convivencia.

Dificulta para hacer amigo

Aburrido

Es todo difícil.

Difícil hablar,

Volver a la primaria. (alumnos de 6 to y 7 mo grado)

Ante la nueva situación escolar (otra escuela, otro nivel educativo) su imagen de sí como aprendientes se encuentra conmovida, interpelada. La imagen de sí que le devuelve su porvenir está signada por la dificultad para aprender (Ia tarea, para hablar), la angustia (miedo, Ilanto), la

16 Soledad Vercellino, Nora Liliana Tarruella, Romina Alejandra van den Heuvel, Rocío Belén Andrade, Mariana Andrea Guerreiro, Cristina Irigoyen y Lidia Mónica Cardinale 
inhibición para hacer lazo con otros (vergüenza, timidez). El otro escolar -virtual, ese 'fantasma del otro' que cada quien lleva dentro de sí (Charlot, 2008)- 'mira mal,' 'se ríe,' 'molesta'. Ante ello, las alternativas son regresivas: volver a la primaria, encontrar conocidos.

Esta conmoción identitaria responde tanto al tiempo de pasaje - de un nivel escolar a otro, de la infancia a la adolescencia- como a la construcción singular de sí como aprendientes, en esa escuela, en ese barrio, en este tiempo histórico.

\section{Conclusiones}

Hasta aquí presentamos algunos resultados de una investigación que analiza cómo el tránsito de ciertos niños y niñas por la institución escolar -con sus propios reservorios de significaciones, lógicas de funcionamiento y demandas hacia el sujeto- constituye su relación con el saber.

La propuesta metodológica desarrollada constituyó una oportunidad para recuperar a partir del discurso oral, escrito y otras expresiones (gráficas y dramáticas) la singularidad de la relación de este alumnado con el saber.

En primer lugar, dimos cuenta de la relación epistémica con el saber que mantiene el alumnado, es decir, identificamos con qué tipos de saberes estos interactúan y que procesos de aprendizajes se dan con estos mismos. Advertimos que este alumnado y su contexto escolar jerarquizan como saber fundamental -y condición para la enseñanza de otros saberes- el dominio de las relaciones intersubjetivas en situación: es decir, saberes morales, políticos sobre como vincularse con los otros sujetos y consigo en el ámbito escolar y no escolar.

Ahora bien, la escuela promueve con estos saberes un modo de relación que se ajusta a lo que Charlot (2008) ha tipificado como "objetivación-denominación". Esto significa que la relación con los saberes relacionales que dispone la escuela lejos de propiciar el dominio, el saber conducirse en situación, el saber actuar, instala al saber relacional en la figura de un saber-objeto, es decir, lo convierte en enunciados normativos sobre el buen conducirse y la relación con las demás personas. El saber relacional aparece, entonces, como "un existente en sí, en un universo de saberes distinto del mundo de la acción, de las percepciones, de las emociones" (Charlot, 2008, p. 112) que es donde se aprende a relacionarse con los otros seres y consigo mismo o misma.

También observamos que el alumnado domina ciertas actividades propias de lo escolar (la escritura, la tecnología): pero lo hacen bajo cánones, criterios, normatividades no escolares. La escritura no responde a la lengua estándar, el uso de las TIC no es el previsto.

En este punto, ante el desafío planteado por organismos gubernamentales y académicos respecto de lograr que el alumnado se apropie del conjunto de conocimientos que el sistema educativo ha seleccionado y prevé transmitirle (Poggi, 2015), nuestra investigación se pregunta ¿la adquisición de qué tipos de saberes (objetos o conocimientos, dominios relacionales o de 
doi: http://dx.doi.org/10.15359/ree.21-3.10

URL: http://www.una.ac.cr/educare

CORREO: educare@una.cr

actividad) promueven las figuras del aprender que propone la escuela? ¿Resultan pertinentes dichas figuras para adquirir los saberes escolares que pretenden enseñar y acreditar?

Estas preguntas permiten correr el foco del déficit del alumnado a la potencialidad de la intervención de la escuela; reconocer que la problemática se ubica no en la ausencia de oferta, sino en la necesidad de repensarla en término de las relaciones con el saber que esta promueve; ubicar la centralidad de las figuras del aprender que, más o menos reflexivamente, se promueven en la escuela y analizar que figuras del aprender se han instituido y cómo ellas mismas conspiran contra la posibilidad de aprender.

Esta investigación permite interrogar, asimismo, el diagnóstico que señala que el alumnado establece con la escuela y con sus aprendizajes relaciones de baja intensidad (Kessler, 2007). Por el contrario, este alumnado solo identifica a la escuela y a su familia como los lugares donde se aprende y en dónde se encuentran las personas adultas referentes del aprender. Este estudiantado vive con intensidad su escolaridad (pasada, presente y futura), al punto que, por ejemplo el pasaje al nivel medio los conmueve e interpela. La imagen de sí que le devuelve su porvenir está signada por la dificultad, la angustia y una demanda de hospitalidad hacia el nuevo espacio escolar.

Pesquisar la relaciones con el saber que el alumnado reconoce como posibilitadoras, de gran valor e interés aún cuando se dan en ámbitos no escolares (como lo son para quienes participaron de esta investigación, el futbol, la música, otros deportes), da pistas de las condiciones necesarias para promover otras relaciones con el saber escolar: ser convocante en "mi mundo" con "otros"; ser un saber hacer de gran valor en la comunidad, ser una vía de reconocimiento, de identificación, ser un significante que hace lazo social.

\section{Referencias}

Anderson-Levitt, K. M. (Ed.). (2012). Anthropologies of education: A global guide to ethnographic studies of learning and schooling. New York: Berghahn Books.

Andrade R. B. (2016) ¿Qué relación con el saber construyen adolescentes mapuches en secundarios insertos en comunidades mapuches? (Tesis de pregrado). Universidad Nacional del Comahue-CURZA, Viedma.

Beillerot, J., Blanchard-Laville, C. y Mosconi, N. (1998). Saber y relación con el saber. Buenos Aires: Paidos Educador.

Camilloni, A. (2001). Modalidades y proyectos de cambio curricular. En N. Schuster (Ed.), Aportes para un cambio curricular en argentina 2001 (pp. 23-52). Buenos Aires: UBA y OPS. Recuperado de http://publicaciones.ops.org.ar/publicaciones/politicas sist servicios/ Aportes\%20para\%20un\%20cambio\%20curricular.pdf

18 Soledad Vercellino, Nora Liliana Tarruella, Romina Alejandra van den Heuvel, Rocío Belén Andrade, Mariana Andrea Guerreiro, Cristina Irigoyen y Lidia Mónica Cardinale

Los artículos de la Revista Electrónica Educare del Centro de Investigación y Docencia en Educación de la Universidad Nacional, Costa Rica, se comparten bajo términos de la Licencia Creative Commons: Reconocimiento, № Comercial, Sin Obra Derivada 3.0 Costa Rica. Las autorizaciones adicionales a las aquí delimitadas se pueden obtener en el correo: educare@una.cr 
Cardinale, L. (2015). Escuelas de jornada extendida: ¿Conveniencia u oportunidad? Revista latinoamericana de educación inclusiva, 9(1), 95-109. Recuperado de https://dialnet. unirioja.es/servlet/articulo?codigo $=5155492$

Cardinale, L. (2016). Límites y efectos de nuevos espacios curriculares en la construcción de una escuela más justa: El caso de las escuelas de jornada extendida de la Ciudad de Viedma (Tesis de maestría). Universidad Nacional del Centro de la Provincia de Buenos Aires, Buenos Aires, Tandil.

Cardinale, L. y Abel, A. N. (2013). La pregunta por la contención. Pilquen. Sección Psicopedagogía, 15(10), 1-8. Recuperado de https://dialnet.unirioja.es/servlet/articulo?codigo=4690699

Charlot, B. (2008). La relación con el saber. Elementos para una teoría. Buenos Aires: Libros del Zorzal. Chevallard, Y. (2000). La transposición didáctica. Del saber sabio al saber enseñado. Buenos Aires: Aique.

Glaser, B. G. y Strauss, A. L. (2009). The discovery of grounded theory: Strategies for qualitative research. Londres: Transaction Publishers. Recuperado de http://www.sxf.uevora.pt/wpcontent/uploads/2013/03/Glaser 1967.pdf

Gobierno de Río Negro. Ministerio de Educación y Derechos Humanos. (2015). Estadísticas Educativas Anuario educativo 2015. Viedma, Río Negro, Ministerio de Educación. Recuperado de http://www.campus1.rionegro.gov.ar/descargar.php?id=7284

Guerreiro, M. (2016). La relación de sujetos adultos con el saber corporal en el marco de la enseñanza y el aprendizaje del método Pilates (Tesis de pregrado). Universidad Nacional del Comahue, Centro Universitario Regional Zona Atlántica, Viedma.

Kalali, F. (2007). Rapport au savoir: Bilan sur la place du sujet dans les différents travaux. Symposium: Rapports au (x) savoir(s): du concept aux usages. Congrès international AREF (Actualité de la recherche en education et en formation). Strasbourg. Recuperado de http://www.congresintaref.org/actes pdf/AREF2007 Faouzia KALALI 422.pdf

Kessler, G. (2007). Escuela y delito juvenil. La experiencia educativa de jóvenes en conflicot con la ley. Revista Mexicana de Investigación Educativa, 12(032), 283-303. Recuperado de http:// www.comie.org.mx/v1/revista/visualizador.php?articulo=ART32014\&criterio=http:// www.comie.org.mx/documentos/rmie/v12/n032/pdf/N032N.pdf

Mar, H. (2009). Pedagogía y arquitectura [Editorial]. Educación y Pedagogía, 21(54), 5-8. Recuperado de http://aprendeenlinea.udea.edu.co/revistas/index.php/revistaeyp/ article/view/9777/8986 
doi: http://dx.doi.org/10.15359/ree.21-3.10

URL: http://www.una.ac.cr/educare

CORREO: educare@una.cr

Ministerio de Educación y Deportes de la Nación. Dirección Nacional de Información y Estadística Educativa (DINIEE). (2015). Porcentaje de alumnos con sobreedad 2015 por nivel educativo según división político-territorial. Ciudad Autónoma de Buenos Aires, Dirección Nacional de Información y Estadística Educativa. Recuperado de http://portales.educacion.gov.ar/ diniece/indicadores-educativos/

Poggi, M. (2015). Introducción. En M. Poggi (Coord.), Mejorar los aprendizajes en la educación obligatoria: Políticas y actores (pp. 11-14). Ciudad Autónoma de Buenos Aires: Instituto Internacional de Planeamiento de la Educación IIPE-Unesco. Recuperado de http:// unesdoc.unesco.org/images/0023/002349/234977s.pdf

Pouliot, C., Bader, B.y Therriault, G. (2010). The notion of the relationship to knowledge: A theoretical tool for research in science education. International Journal of Environmental \& Science Education, 5(3), 239-264. Recuperado de http://files.eric.ed.gov/fulltext/EJ895738.pdf

Quilaqueo, D., Quintriqueo, S.y Cárdenas, P.(Eds.). (2005). Educación, currículum einterculturalidad: Elementos sobre formación de profesores en contexto mapuche. Temuco: Universidad Católica de Temuco.

Quintriqueo, S. y Torres, H. (2012). Distancia entre el conocimiento mapuche y el conocimiento escolar en contexto mapuche. Revista Electrónica de Investigación Educativa, 14(1), 16-33. Recuperado de http://www.redalyc.org/pdf/155/15523175002.pdf

Rochex, J.-Y. (2004). La notion de rapport au savoir: Convergences et débats théoriques. Pratiques Psychologiques, 10(2), 93-106. doi: https://doi.org/10.1016/j.prps.2004.03.001

Scasso, L. M. (2012). La educación en cifras. Indicadores seleccionados para la caracterización del sistema educativo Río Negro. Buenos Aires: UNICEF. Recuperado de https://www.unicef. org/argentina/spanish/rio negro.pdf

Thériault, G., Bader, B. y Ndong Angoe, C. (2013). L'apport de la notion de rapport(s) au(x) savoir(s) en education aux sciences et en formation initiale et continue des enseignants du secondaire: Des exemples au Québec et au Gabon. Revue Esprit critique, 17, 70-94.

Universidad Nacional del Comahue. Departamento de Psicopedagogía. (2013). Relación de los alumnos con el saber y formato escolar. Investigación teórica y empírica con alumnos de escuelas primarias de la ciudad de Viedma, Río Negro. 2013-2014 (PI V04-084). Viedma, Río Negro: Autor.

Universidad Nacional del Comahue. Departamento de Psicopedagogía. (2015). La constitución de la relación con el saber en los alumnos del último ciclo del nivel primario y primero del nivel medio. Viedma, Río Negro. 2015-2016 (PI V097). Viedma, Río negro: Autor.

20 Soledad Vercellino, Nora Liliana Tarruella, Romina Alejandra van den Heuvel, Rocío Belén Andrade, Mariana Andrea Guerreiro, Cristina Irigoyen y Lidia Mónica Cardinale

Los artículos de la Revista Electrónica Educare del Centro de Investigación y Docencia en Educación de la Universidad Nacional, Costa Rica, se comparten bajo términos de la Licencia Creative Commons: Reconocimiento, № Comercial, Sin Obra Derivada 3.0 Costa Rica. Las autorizaciones adicionales a las aquí delimitadas se pueden obtener en el correo: educare@una.cr 
van den Heuvel, R. (2016). Movimientos conceptuales en el campo psicopedagógico. Análisis de las relaciones entre conceptos propuestos por Jacky Beillerot y Alicia Fernández (Tesis de pregrado). Universidad Nacional del Comahue, Centro Universitario Regional Zona Atlántica, Viedma.

van den Heuvel, R. y Vercelino, S. (2015).La movilidad de conceptos en el campo psicopedagógico: Una aproximación a las relaciones entre conceptos propuestos por Jacky Beillerot y Alicia Fernández. Revista Pilquen. Sección Psicopedagogía, 12(1), 1-13. Recuperado de http:// www.curza.net/revistapilquen/index.php/Psico/article/view/59

Venturini, P., Calmettes, B., Amade-Escot, C. y Terrisse, A. (2007). Analyse didactique des practieus d' enseignement de la physique d'un professeure experimentée. Aster, 45, 211-234. Recuperado de https://doi.org/10.4267/2042/16824

Vercellino, S. (2013). La (re)organización del dispositivo escolar en escuelas primarias de la provincia de Río Negro que implementan un programa de extensión de la jornada escolar. Viedma: Universidad Nacional de Río Negro.

Vercellino, S. (2014). La 'relación con el saber': Revisitando los comienzos del concepto. Revista Pilquen. Sección Psicopedagogía, 16(11), 1-8. Recuperado de http://www.revistapilquen. com.ar/Psicopedagogia/Psico11/11 6Vercellino Relacion.pdf

Vercellino, S. (2015). Revisión bibliográfica sobre la 'relación con el saber'. Desplazamientos teóricos y posibilidades para el análisis psicopedagógico de los aprendizajes escolares. Revista Electrónica Educare, 19(2), 53-82. doi: https://doi.org/10.15359/ree.19-2.4

Vercellino, S., van den Heuvel, R., Guerreiro, M. (2014). Deslocamentos teóricos da noção da "Relação com o saber" e suas possibilidades para a análise psicopedagógica das aprendizagens escolares". Psicopedagogia, 31(96), 275-288. Recuperado de http://pepsic. bvsalud.org/pdf/psicoped/v31n96/05.pdf

Zambrano, A. (2013). Relación con el saber, fracaso/éxito escolar y estrategias de enseñanzaaprendizaje. Actualidades Pedagógicas, 61, 27-43. doi: https://doi.org/10.19052/ap.2329 